\title{
An integrated 3.1-5.1 GHz pulse generator for ultra-wideband wireless localization systems
}

\author{
X. Fan, G. Fischer, and B. Dietrich \\ IHP, Im Technologiepark 25, D-15236 Frankfurt (Oder), Germany
}

\begin{abstract}
This paper presents an implementation of an integrated Ultra-wideband (UWB), Binary-Phase Shift Keying (BPSK) Gaussian modulated pulse generator. VCO, multiplier and passive Gaussian filter are the key components. The VCO provides the carrier frequency of $4.1 \mathrm{GHz}$, the LC Gaussian filter is responsible for the pulse shaping in the baseband. Multiplying the baseband pulse and the VCO frequency shifts the pulse to the desired center frequency. The generated Gaussian pulse ocupppies the frequency range from 3.1 to $5.1 \mathrm{GHz}$ with the center frequency at $4.1 \mathrm{GHz}$. Simulations and measured results show that this spectrum fulfills the mask for indoor communication systems given by the FCC (Federal Communications Commission, 2002). The total power consumption is $55 \mathrm{~mW}$ using a supply voltage of $2.5 \mathrm{~V}$. Circuits are realized using the IHP $0.25 \mu \mathrm{m} \mathrm{SiGe:C}$ BiCMOS technology.
\end{abstract}

\section{Introduction}

Ultra-wideband is seen as one of the promising technologies for short range and high speed network and position localization applications. Since the Federal Communications Commission (FCC) rulings were released in February 2002, the design and implementation of UWB systems have received more and more attention. Up to now, a number of proposals for UWB systems were already submitted. For the UWB high data rate (HDR) systems, which mainly focus on communication application, two proposals are still left in competition. But for the low data rate (LDR) UWB systems the pulse based UWB proposal is already accepted and the 802.15.4a task group is now working on it. The group's main task is to achieve a system with a high precision ranging/localization capability ( $1 \mathrm{~m}$ accuracy or even better). Relating to this a critical issue in the design of the

Correspondence to: Xi Fan (fan@ihp-microelectronics.com)

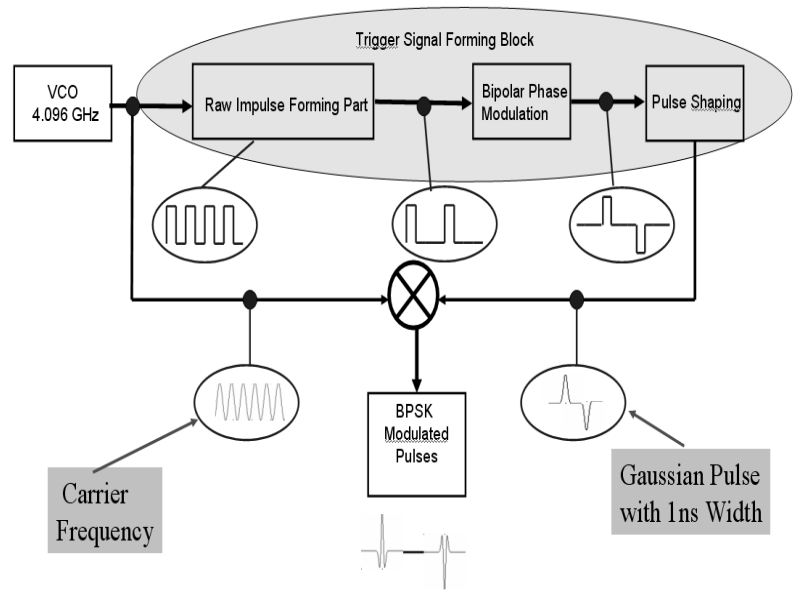

Fig. 1. Principle of carrier based pulse generator.

pulsed UWB transceiver is an integrated UWB pulse generator, which fulfills the spectrum mask defined by the FCC.

Many UWB pulse generators have been introduced. Most of them either can not be completely integrated in Sitechnology (Marsden et al., 2003) or the center frequency of the pulse can not be flexibly controlled (Gerrits and Farserotu, 2001) or they need special semiconductor device (Han et al., 2002). A pulse generator using two independent sources, one oscillator for the center frequency (carrier frequency) and one generator for the baseband pulse, is reported in Harbin and Lawrence (2001). In this work an alternative solution for the pulse generator is presented, where the baseband pulse frequency is derived from the carrier frequency. Its main advantage is the jitter-independence in addition to the flexibility of moving center frequency. That means the jitter caused by the oscillator will not influence the generated pulse shape, which is not the case in Harbin and Lawrence (2001). This pulse generator is designed for

Published by Copernicus GmbH on behalf of the URSI Landesausschuss in der Bundesrepublik Deutschland e.V. 


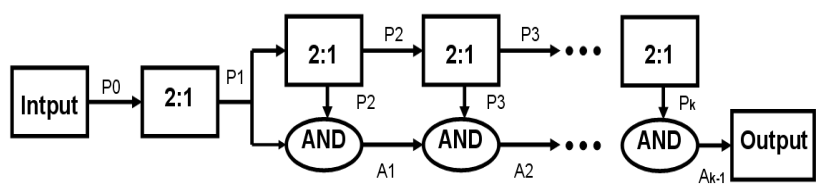

Fig. 2. Generation of raw pulse train.

the frequency range from 3.1 to $5.1 \mathrm{GHz}$. The choice of this frequency range makes technology a lot easier, fits with the lower bound of the FCC ruling and avoids the WLAN frequency range $(5.1 \mathrm{GHz})$. The block diagram of this pulse generator is shown in Fig. 1.

The VCO is designed to provide $4.1 \mathrm{GHz}$ carrier frequency. The output of the VCO is fed into the multiplier over two paths. One goes directly into the first input of the multiplier and the other goes into the second input after passing a trigger signal forming block. The trigger signal forming block consists of the raw impulse forming part, the BPSK modulator and the pulse shaping filter, which are described in the next sections respectively. The raw impulse forming part consists of $k$ 2:1 dividers which gives a total division ratio $2^{k}: 1$. Each divider is followed by an ECL AND-gate (Figs. 2 and 3). The outputs of the dividers generate sequentially the Boolean AND functions of all previous operands accomplishing $k-1$ AND operations. After that a raw impulse train appears at the output of the raw impulse forming part with the pulse width

$W_{\text {pulse }}=\frac{1}{f_{\mathrm{vco}}}, \quad f_{\mathrm{vco}}, \mathrm{VCO}$ frequency

and the duty cycle of

$D_{\text {cycle }}=\frac{100}{2^{k}} \%$

This rectangle pulse train is then BPSK modulated and triggers the following pulse shaping filter to generate the baseband Gaussian pulses, which are then fed into the second input of the multiplier. At the output of the multiplier the Gaussian shaped pulses with the center frequency of $4.1 \mathrm{GHz}$ appear. In this work the VCO designed in Herzel et al. (2003) using the IHP SiGe:C BiCMOS technology is taken over. So it is not described here again.

In the following sections we firstly describe the key components used in this design. And then the simulation and measurement results are shown. Finally a conclusion is given.

\section{Raw impulse forming}

The raw impulse forming part should be able to generate raw impulse to trigger the pulse forming filter. The name raw

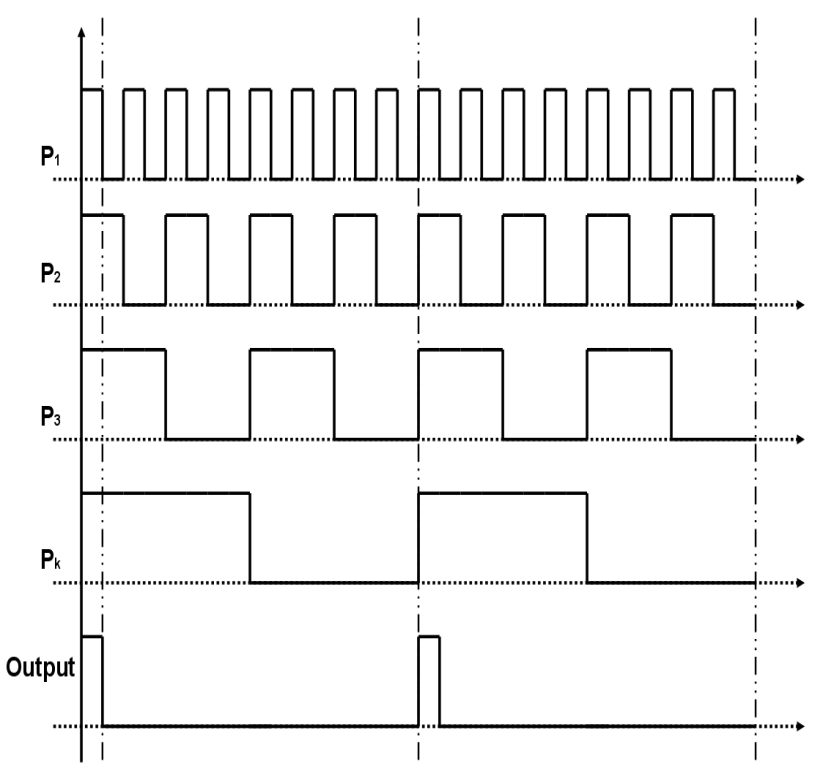

Fig. 3. Generation of raw pulse train: waveform.

used here represents un-shaped impulses in order to distinguish them from the shaped pulses after the shaping filter. The ideal raw impulse form is Dirac impulse, which is practically not realizable. A possible way is to use another raw impulse form to approximate the Dirac impulse. Rectangle, triangle, exponential pulses are common used raw impulse forms. In our case, we use the simple one, the rectangle impulse form. To generate them we combine the bipolar frequency dividers with ECL AND-gates to build a chain (Fig. 2). The chain length is determined by the division ratio. Figure 3 shows the timing diagram. One can see from the timing diagram that the output pulse width is defined by the pulse width of rectangle signal with the highest frequency (1). The period of the output pulses depends on the rectangle signal with the lowest frequency (2). For measurement purpose we choose the division ratio 32:1. That means we need five 2:1 dividers and four ECL AND-gates.

The use of rectangle pulses as an approximation for Dirac impulses requires sharp impulse edge. Furthermore such pulse generators should not be considered only for lower band 3.1-5.1 GHz, but also for higher band, for example 7.2$9.2 \mathrm{GHz}$, within the FCC mask. Due to the higher transit frequency bipolar transistors are chosen for the implementation of gigahertz Circuits. Using the bipolar transistors of the IHP SiGe:C BiCMOS process we achieve rectangle pulse with about $50 \mathrm{ps}$ rise and fall time.

\section{Modulation, pulse shaping filter and multiplier}

Different from the common way the impulse train is modulated with the data stream before the pulse shaping filter. 


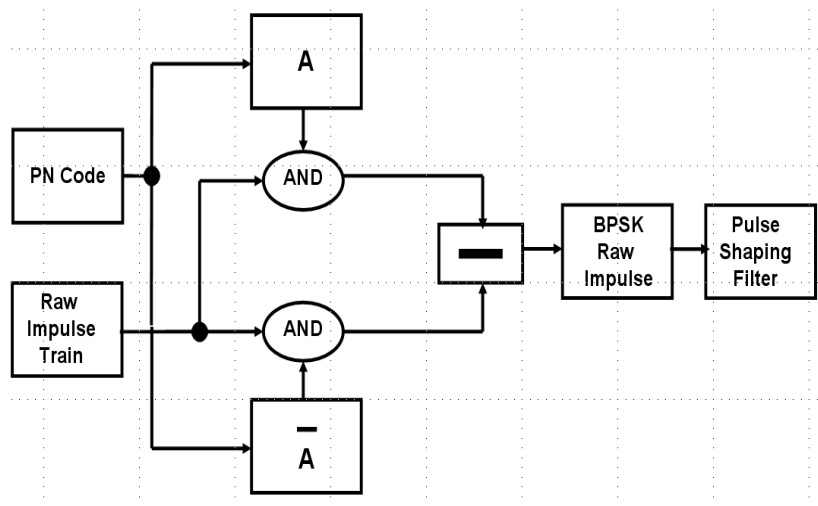

Fig. 4. Principle of BPSK modulation.

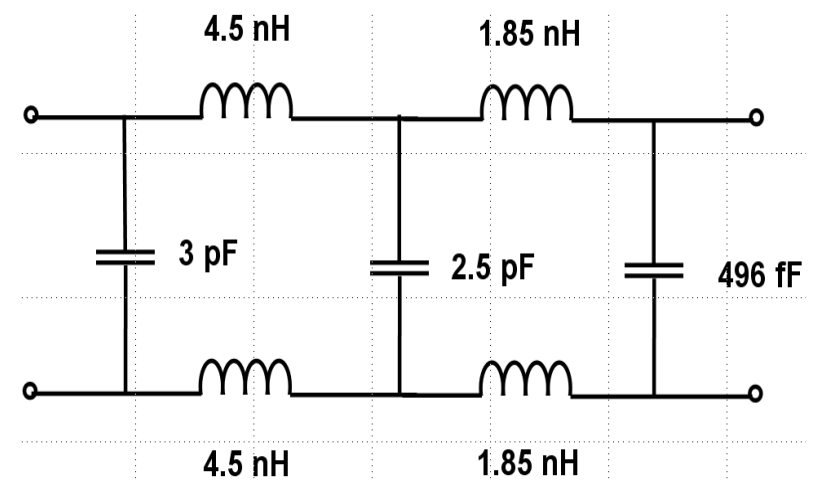

Fig. 5. Passive 5th Order LC Gaussian filter.

That means the generated raw impulse train is firstly BPSK modulated, then the modulated raw impulse train triggers the pulse shaping filter. This principle is illustrated in Fig. 4. For simulation two complemental pseudo-noise (PN) codes are derived from a PN code source. The complementary PN codes are then added to the same raw impulse train respectively. The outputs of both ECL AND-gates are then subtracted to build the desired BPSK modulated raw impulse train. This BPSK modulated impulse train is then used to trigger the following pulse shaping filter.

The pulse shaping filter is responsible for shaping very short baseband pulses. Such pulses are desired in the pulse based UWB systems because they can reduce the intersymbol-interference (ISI), increase robustness against multipath fading and allow episodic transmission for saving the power. One shaping possibility is using the Gaussian filter. Due to the very short pulse duration, $1 \mathrm{~ns}$ in our case, a solution using active filter could be a very difficult task. Therefore a passive solution, i.e. using a passive LC Gaussian filter, is preferred. The main disadvantage is the occupation of large chip area. In this work we choose the passive solution since we firstly want to show the functionality of the pulse generator. The Fig. 5 shows a differential baseband Gaussian
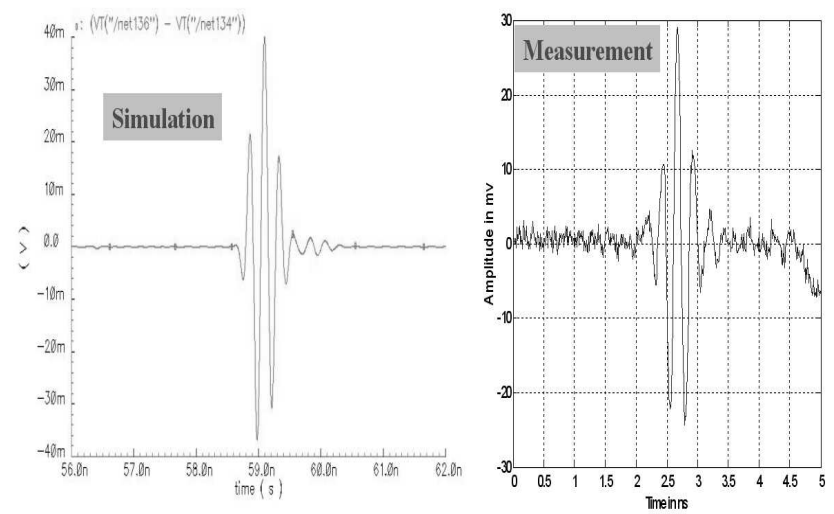

Fig. 6. Pulse in the time domain.
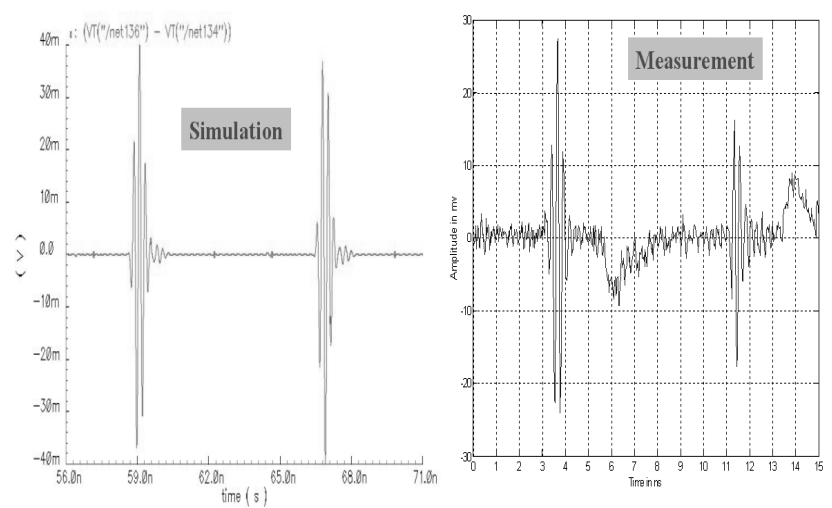

Fig. 7. BPSK modulated pulses.

filter of order 5 used in this work. In the layout the top metal layer is used for the inductor design to increase the Q-factor.

The common Gilbert Mixer is used as multiplier to multiply the carrier and baseband Gaussian pulses. Because of its small linear range both input signals should be kept small so that higher harmonics could be avoided. At the output of the mixer several amplifier stages can be cascaded to get the desired output power.

\section{Simulation and measurement results}

To get better comparison of the simulation and measurement results every plot in this section is separated in two sub-plots serially. The left one is the simulation result and the right one is the result of the measurement. In Fig. 6 the simulation shows an individual pulse at the output of the multiplier. It has pulse width of $1 \mathrm{~ns}$ and around $80 \mathrm{mV}$ peak-to-peak voltage $\left(V_{p p}\right)$. The measured result matches nearly the simulation except small reduction of $V_{p p}$.

The Fig. 7 gives a pair of BPSK modulated pulses at the output of the multiplier. We can see that these pulses are 


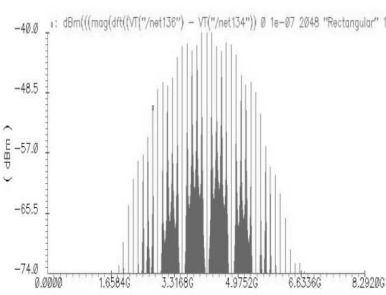

Simulation

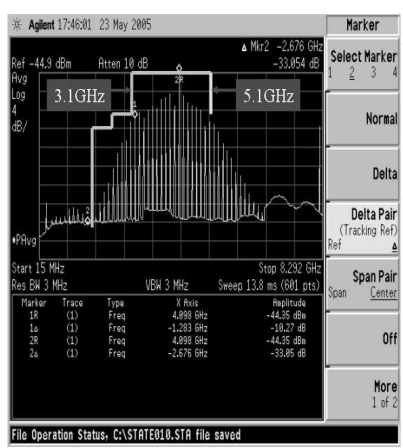

Measurement

Fig. 8. Pulse in the frequency domain.

kept in $8 \mathrm{~ns}$ distance and have 180 degree phase shift to each other both in the simulated and measured results. But in the measured results the second pulse is obviously smaller than the first one. And one spurious peak also occurs between one pulse pair. These could be caused by the mismatch in the process and too small blocking capacitors used in the layout.

Up to now we discussed the results in the time domain. The spectrum of such pulses should also be investigated to see weather it fulfills the FCC mask. Figure 8 shows the spectra of the simulation and measurement. Here they match each other quite well. Limited by the measurement condition we use a periodic rectangle signal instead of the PN code. This periodicity is then delivered to the pulses at the output of the multiplier, which results in the spectral lines in the spectrum of the generated pulses. The frequency span between spectral lines in this case is $125 \mathrm{MHz}$, which corresponds to $8 \mathrm{~ns}$ time distance in the time domain (Fig. 7). Such spectral lines will then become smooth, if the PN code is applied. It has to be pointed out that these results both in the time and frequency domain are obtained without UWB antenna. With the antenna a little modification of these results in both domains will be expected. However the spectrum of the pulses will still be kept in the FCC mask.

\section{Conclusions}

In this paper a Gaussian modulated pulse generator with frequency range from 3.1 to $5.1 \mathrm{GHz}$ for the UWB LDR application is demonstrated. It uses an approximated Dirac impulse for triggering the Gaussian pulse shaping filter and generating the Gaussian pulses in the baseband. Such baseband Gaussian pulses are then multiplied with the carrier sinusoidal signal to generate final pulses. These pulses, holding the baseband Gaussian pulse envelope, carrying the desired center frequency, fulfil the requirement both in the time and frequency domain. Due to the flexibility of this approach a pulse generator for the higher band can be designed just by increasing the carrier frequency to the desired value. The presented solution exhibits a fixed phase relation between the carrier signal and the baseband Gaussian pulses. Therefore it is insensible to the jitter caused by the VCO. This sub-band pulse generator has been fabricated using the IHP $0.25 \mu \mathrm{m}$ BiCMOS in SiGe:C technology.

\section{References}

Federal Communications Commission: First Report and Order, FCC 02-48, 22 April, 2002.

Gerrits, J. F. M. and Farserotu, J. R.: Wavelet generation circuit for UWB impulse radio application, in Electronics Letters, 38, 25, 1737-1738, 2001

Han, J. and Nguyen, C.: A New Ultra-Wideband, Ultra-Short Monocycle Pulse Generator with Reduced Ringing, in IEEE Microwave and Wireless Components Letters, 12, 6, 206-208, 2002.

Harbin and Lawrence: Ultra-Wideband Data Transmission System, in Pub.No.: WO01/39451 A1, 2001.

Herzel, F., Winkler, W., and Borngrber, J.: An Integrated $10 \mathrm{GHz}$ Quadrature LC-VCO in SiGe:C BiCMOS Technology for LowJitter Applications, in Proc. IEEE Custom Integrated Circuits Conf. (CICC), San Jos, 293-296, 2003.

Greub, H. J., McDonald, J. F., Greedon, T., and Yamaguchi, T.: High-Performance Standard Cell Library and Modeling Technique for Differential Advanced Bipolar Current Tree Logi, in IEEE Journal of solid-state circuits, 26, 5, 749-762, 1991.

Marsden, K., Lee, H.-J., Ha, D. S., and Lee, H.-S.: Low Power CMOS Re-programmable Pulse Generator for UWB System, in Int. Conf. on Ultra Wideband Systems and Technologies, Reston,Virginia, 443-447, 2003. 